\title{
Magnetic shear-driven instability and turbulent mixing in magnetized protostellar disks
}

\author{
A. Bonanno ${ }^{1,2}$ and V. Urpin ${ }^{1,3}$ \\ 1 INAF - Osservatorio Astrofisico di Catania, via S. Sofia 78, 95123 Catania, Italy \\ e-mail: abo@oact.inaf.it \\ 2 INFN, Sezione di Catania, via S. Sofia 72, 95123 Catania, Italy \\ 3 A.F. Ioffe Institute of Physics and Technology and Isaac Newton Institute of Chile, Branch in St. Petersburg, 194021 St. Petersburg, \\ Russia
}

Received 28 March 2007 / Accepted 5 December 2007

\section{ABSTRACT}

\begin{abstract}
Context. Observations of protostellar disks indicate the presence of the magnetic field of thermal (or superthermal) strength. In such a strong magnetic field, many MHD instabilities responsible for turbulent transport of the angular momentum are suppressed. Aims. We consider the shear-driven instability that can occur in protostellar disks even if the field is superthermal.

Methods. This instability is caused by the combined influence of shear and compressibility in a magnetized gas and can be an efficient mechanism to generate turbulence in disks.

Results. The typical growth time is of the order of several rotation periods.
\end{abstract}

Key words. accretion, accretion disks - magnetohydrodynamics (MHD) - instabilities - turbulence - stars: formation

\section{Introduction}

Protostellar disks require sufficiently strong turbulence to enhance the efficiency of angular momentum transport. The origin of turbulence is often attributed to hydrodynamic and hydromagnetic instabilities that can arise in differentially-rotating, stratified gaseous disks. One of the candidates is magnetorotational instability (MRI), which can operate in a conductive flow if the angular velocity decreases with the cylindrical radius and the magnetic field is not strong (Velikhov 1959). The MRI has been studied in depth in the case of stellar and accretion disk conditions (see, e.g., Fricke 1969; Safronov 1969; Acheson 1978, 1979; Balbus \& Hawley 1991; Kaisig et al. 1992; Kumar et al. 1994; Zhang et al. 1994). Simulations of the MRI in disks (Hawley et al. 1995; Matsumoto \& Tajima 1995; Brandenburg et al. 1995; Torkelsson et al. 1996; Arlt \& Rüdiger 2001) show the turbulence generated can significantly enhance the angular momentum transport.

Most likely, however, the number of instabilities that can arise in astrophysical disks is quite large. An analysis of MHD modes in stratified accretion disks demonstrates a wide variety of instabilities even in the case of simple magnetic geometry (Keppens et al. 2002). Therefore, the current point of view on the origin of turbulence in disks is likely highly simplified. Even pure, hydrodynamic origin of turbulence cannot be excluded (see Urpin 2003; Arlt \& Urpin 2004; Dubrulle et al. 2005) despite the most efficient local linear hydrodynamic instabilities advocated to date are not sufficiently efficient. Global instabilities, such as the baroclinic-like instability of Klahr \& Bodenheimer (2003), are sensitive to boundary conditions (Johnson \& Gammie 2006) and, therefore, are unlikely to drive turbulence in disks. Note, however, that such factors as fast radiative cooling, high thermal diffusion, and large radial temperature gradients can strengthen the baroclinic feedback and make the baroclinic instability more efficient (Petersen et al. 2007). Astrophysical disks are stratified and stratification can change stability properties of shear flows, providing either a stabilizing or destabilizing effect, depending on details of the disk structure. Recently, Dubrulle et al. (2005) have considered one of the possible "strato-rotational" instabilities arising in the presence of both differential rotation and stable stratification. However, Brandenburg \& Rüdiger (2005) demonstrated that the growth rate of this instability decreases with an increasing Reynolds number, rendering the instability less relevant for astrophysical applications. Note also that convection can drive turbulence in disks with unstable stratification, but it induces inward turbulent transport of the angular momentum instead of the required outward one (see, e.g., Stone \& Balbus 1996).

As it has been argued by Lesur \& Longaretti (2005), nonlinear hydrodynamic instability that often occurs in linearly-stable flows at sufficiently large Reynolds numbers is likely inefficient in disks even for the most optimistic extrapolations of numerical data. At least, the subcritical transition to hydrodynamic turbulence cannot occur in quasi-Keplerian flows at Reynolds numbers up to $\sim 10^{6}$ ( $\mathrm{Ji}$ et al. 2006). Of course, Reynolds numbers are much higher in real disks; nevertheless it seems that there is little room for nonlinear instability.

The stability properties of protostellar disks are much different from those of accretion disks. The magnetic Reynolds number is likely not very large in cold- and dense-protostellar disks because of a low electrical conductivity, and so the field cannot be treated as "frozen" into the gas (Gammie 1996). The effect of Ohmic dissipation on the MRI has been considered in the linear (Jin 1996) and nonlinear regimes (Sano et al. 1998; Drecker et al. 2000). Fleming \& Stone (2003) and Turner et al. (2006) treated turbulent mixing caused by the MRI in protostellar disks, taking into account magnetic diffusivity. They found that the midplane is shielded from cosmic rays, and that MRI does not occur 
even under the most favorable conditions. Nevertheless, turbulence can mix a fraction of the weakly-conducting surface material into the interior, providing some coupling of the midplane gas to the magnetic field. As a result, weak stresses can appear in the disk midplane.

As first pointed out by Wardle (1999), poorly conducting protostellar disks can be strongly magnetized if electrons are the main charge carriers. Therefore, transport must be anisotropic with substantially different properties along and across the magnetic field. In strongly magnetized gas the Hall component provides the main contribution to the resistivity tensor, which produces the electric field perpendicular to both the magnetic and electric current. The stability analysis by Wardle (1999) shows that the Hall effect can provide either stabilizing or destabilizing influences depending on the direction of the field. Balbus \& Terquem (2001) have conducted a more general study of the role of the Hall term on MRI. They found that the Hall effect qualitatively changes the stability properties of protostellar disks and can lead to instability even if the angular velocity increases outward. These authors, however, did not take into account the effect of gravity that is crucial for disks. Urpin \& Rüdiger (2005) considered MRI under the combined influence of the Hall effect and gravity, and also derived also the criteria of several other instabilities that can occur in protostellar disks. Sano \& Stone (2002) investigated the effect of the Hall term on the evolution of the MRI in weakly-ionized disks using local axisymmetric simulations. These authors concluded the properties of the MRI depend essentially on the direction of the magnetic field as it is anticipated from the dispersion equation in a linear stability analysis. Salmeron \& Wardle (2005) have also considered the properties of the MRI modified by the Hall effect. These authors argued that the MRI is active in protoplanetary disks over a wide range of field strengths and fluid conditions. The Hall conductivity results in a faster growth of perturbations and extends the region of instability. Recently, Livertz et al. (2007) and Shtemler et al. (2007) have considered the Hall MRI in a non-axisymmetric case. This type of instability is proposed as a viable mechanism for the azimuthal fragmentation of the protoplanetary disks and planet formation. The non-axisymmetric instability is caused by the combined effect of the radial stratification and Hall electric field. Note that the MRI and its modifications can be completely suppressed if the magnetic field is sufficiently strong (see Urpin 1996; Kitchatinov \& Rüdiger 1997).

If rotation is cylindrical and $\Omega=\Omega(s)$, where $\Omega$ is the angular velocity and $s$ is the cylindrical radius, the critical magnetic field that suppresses the MRI is given approximately by the condition $c_{\mathrm{A}} / H \sim s \Omega^{\prime} \sim \Omega$ where $c_{\mathrm{A}}=B / \sqrt{4 \pi \rho}$ is the Alfvén velocity. Since $\Omega \sim c_{\mathrm{s}} / H$ in the standard disk, where $c_{\mathrm{s}}$ is the sound speed, we find that the MRI is suppressed if the magnetic field is superthermal, $c_{\mathrm{A}}>c_{\mathrm{s}}$. Recent measurements (Hutawarakorn \& Cohen 1999, 2005; Donati et al. 2005) indicate that the magnetic field can be strong in protostellar disks. This particularly concerns the innermost regions where the field strength reaches $\sim 1 \mathrm{kG}$ at the radius $\sim 0.05 \mathrm{AU}$ (Donati et al. 2005). The field configuration includes the azimuthal component whose direction agrees with the radial field sheared by the disk differential rotation. The derived ratio of the azimuthal and radial fields in the surface layers is $\sim 0.5$. Note that the azimuthal component can be substantially stronger than the radial one in the disk interiors because dynamo theories (such as the $\alpha-\Omega$ dynamo) usually predict that the generated field should have a strong toroidal component. According to estimates by Donati et al. (2005), the equipartition field strength with roughly equal thermal and magnetic pressures should be $\sim 10^{3}$ Gauss at the radius $\sim 0.05 \mathrm{AU}$ in protostellar disks. This indicates the detected field is approximately of the thermal or even superthermal strength and can substantially modify or even suppress the MRI.

Recently, Bonanno \& Urpin $(2006,2007)$ have argued that a compressible, differentially-rotating flow is unstable if the magnetic field has a non-vanishing, radial component. A remarkable feature of this shear-driven instability is that it can arise even if the magnetic field is superthermal. Bonanno \& Urpin (2006, 2007) have considered the instability in the case of a highly conductive plasma when the magnetic diffusivity plays no role. However, the instability can also arise in weakly-ionized protostellar disks where the magnetic field has a radial component detected in observations (Donati et al. 2005). The effect of a finite diffusivity can be important for the onset of instability and for the properties of generated turbulence, particularly near the the mid-plane where conductivity is extremely low. In this paper, we consider the shear-driven instability in the conditions of protostellar disks and show that the conditions of instability in weakly and highly ionized plasmas differ substantially. Nevertheless, the shear-driven instability can manifest itself even in weakly-ionized protostellar disks.

The paper is organized as follows. In Sect. 2, we consider the basic equations governing instability in compressible dissipative fluids and we then derive the dispersion relation. In Sect. 3, we derive the criteria of instability and discuss conditions under which instability can occur in protostellar disks. The growth rate of instability is calculated in Sect. 4. Finally, in Sect. 5, we discuss the results obtained.

\section{Basic equations and dispersion relation}

The electrical conductivity is low in protostellar disks- and the magnetic field cannot be considered as "frozen" into the gas. The magnetic diffusivity is given by $\eta=c^{2} m_{\mathrm{e}} / 4 \pi \mathrm{e}^{2} n_{\mathrm{e}} \tau$ where $m_{\mathrm{e}}$ and $n_{\mathrm{e}}$ are the mass and number density of electrons, respectively, and $\tau$ is their relaxation time (see, e.g., Spitzer 1978). In protostellar disks, $\tau$ is determined by the scattering of electrons on neutrals, then $\tau=1 / n\langle\sigma v\rangle$ where $\langle\sigma v\rangle$ is the average product of the cross-section and velocity, and $n$ is the number density of neutrals. Using the fitting expression for $\langle\sigma v\rangle$ obtained by Draine et al. (1983), we have

$\eta=2.34 \times 10^{3} f^{-1} T_{2}^{1 / 2} \mathrm{~cm}^{2} \mathrm{~s}^{-1}$,

where $f=n_{\mathrm{e}} / n$ is the ionization fraction, and $T_{2}=T / 100 \mathrm{~K}$, with $T$ being the temperature.

The magnetic diffusivity can be anisotropic in some regions of protostellar disks (Wardle 1999) because the electron gas is magnetized despite a low temperature. The effect of the magnetic field on transport properties is usually characterized by the magnetization parameter $a_{\mathrm{e}}(B)=\omega_{B} \tau$, where $\omega_{B}=e B / m_{\mathrm{e}} c$ is the gyrofrequency of electrons (see, e.g., Spitzer 1978). Again, using the fitting formula by Draine et al. (1983), we can estimate the magnetization parameter as

$a_{\mathrm{e}}(B) \approx 21 B n_{14}^{-1} T_{2}^{-1 / 2}$

where the magnetic field $B$ is measured in Gauss and $n_{14}=$ $n / 10^{14} \mathrm{~cm}^{-3}$. If $a_{\mathrm{e}}(B)>1$, i.e.

$B>0.048 n_{14} \sqrt{T_{2}}$ Gauss,

then the electron transport is anisotropic and the magnetic diffusivity is represented by a tensor. In plasma of protostellar disks, the difference between components of the magnetic diffusivity parallel and perpendicular to the magnetic field is small 
(see, e.g., Balbus \& Terquem 2001). The Hall component is described as

$a_{\mathrm{e}}(B) \eta=\frac{c B}{4 \pi e n_{\mathrm{e}}}$.

We work in cylindrical coordinates $(s, \varphi, z)$ with the unit vectors $\left(\boldsymbol{e}_{\mathrm{s}}, \boldsymbol{e}_{\varphi}, \boldsymbol{e}_{z}\right)$. The equations of compressible MHD read:

$$
\begin{aligned}
& \dot{\boldsymbol{v}}+(\boldsymbol{v} \cdot \nabla) \boldsymbol{v}=-\frac{\nabla p}{\rho}+\boldsymbol{g}+\frac{1}{4 \pi \rho}(\nabla \times \boldsymbol{B}) \times \boldsymbol{B}, \\
& \dot{\rho}+\nabla \cdot(\rho \boldsymbol{v})=0, \\
& \dot{p}+\boldsymbol{v} \cdot \nabla p+\gamma p \nabla \cdot \boldsymbol{v}=0,
\end{aligned}
$$

$$
\begin{array}{r}
\dot{\boldsymbol{B}}-\nabla \times(\boldsymbol{v} \times \boldsymbol{B})+\frac{c}{4 \pi \mathrm{e}} \nabla \times\left[\frac{1}{n_{\mathrm{e}}}(\nabla \times \boldsymbol{B}) \times \boldsymbol{B}\right] \\
+\eta \nabla \times(\nabla \times \boldsymbol{B})=0,
\end{array}
$$

$\nabla \cdot \boldsymbol{B}=0$.

Our notation is as follows: $\rho$ and $\boldsymbol{v}$ are gas density and velocity, respectively; $p$ is pressure; $\gamma$ is the adiabatic index; and $\boldsymbol{g}$ is gravity. The third term on the 1.h.s. of Eq. (8) describes the Hall effect. We cannot neglect this term because the magnetization parameter $a_{\mathrm{e}}$ is large in protostellar disks. However, in what follows, we will consider a special type of perturbation for which the Hall effect is unimportant.

The basic state on which the stability analysis is performed is assumed to be quasi-stationary with the angular velocity $\Omega=$ $\Omega(s)$ and $\boldsymbol{B} \neq 0$. We assume hydrostatic equilibrium for basic state:

$\frac{\nabla p}{\rho}=\boldsymbol{D}+\frac{1}{4 \pi \rho}(\nabla \times \boldsymbol{B}) \times \boldsymbol{B}, \quad \boldsymbol{D}=\boldsymbol{g}+\Omega^{2} \boldsymbol{s}$.

We consider magnetic configurations where both the radial and azimuthal field components are present. The presence of a radial magnetic field and differential rotation leads to the development of the azimuthal field. In protoplanetary disks, however, the rate of stretching of the azimuthal field from $B_{\mathrm{s}}$ is reduced by ohmic dissipation and Hall effect. Then, Eq. (8) yields the following steady-state condition:

$$
\begin{aligned}
{[\nabla \times(\boldsymbol{v} \times \boldsymbol{B})]_{\varphi}=} & \left\{\nabla \times\left[\frac{c}{4 \pi e n_{\mathrm{e}}}(\nabla \times \boldsymbol{B}) \times \boldsymbol{B}\right]\right\}_{\varphi} \\
& +\eta[\nabla \times(\nabla \times \boldsymbol{B})]_{\varphi} .
\end{aligned}
$$

Assuming that $B_{\varphi}>B_{\mathrm{s}}$, Eq. (11) can be transformed into:

$$
\begin{aligned}
& \frac{c}{4 \pi e}\left\{\frac{\partial}{\partial s}\left(\frac{B_{\varphi}}{n_{\mathrm{e}}} \frac{\partial B_{\varphi}}{\partial z}\right)-\frac{\partial}{\partial z}\left[\frac{B_{\varphi}}{n_{\mathrm{e}}}\left(\frac{\partial B_{\varphi}}{\partial s}+\frac{B_{\varphi}}{s}\right)\right]\right\} \\
& -\eta\left(\Delta-\frac{1}{s^{2}}\right) B_{\varphi}=s \Omega^{\prime} B_{\mathrm{s}} .
\end{aligned}
$$

If the Hall effect is negligible, the first term on the 1.h.s. of this equation is small, and the generated toroidal field is stronger than the radial field by a factor of the order of the magnetic Reynolds number, $\mathrm{Re}_{\mathrm{m}}$,

$B_{\varphi} \sim \operatorname{Re}_{\mathrm{m}} B_{\mathrm{s}}$,

where $\operatorname{Re}_{\mathrm{m}}=s \Omega^{\prime} H^{2} / \eta \sim \Omega H^{2} / \eta$ with $H$ being the halfthickness of the disk (we assume $s \Omega^{\prime} \sim \Omega$ ). If the magnetic field is strong and the Hall effect dominates ohmic dissipation, then one estimates from Eq. (12) that,

$B_{\varphi} \sim \frac{\operatorname{Re}_{\mathrm{m}}^{1 / 2}}{a_{\mathrm{e}}^{1 / 2}\left(B_{\mathrm{s}}\right)}\left(\frac{s}{H}\right)^{1 / 2} B_{\mathrm{s}}$.

In strongly magnetized disks, the generated toroidal field can be substantially weaker than what follows from the simplest estimate (13). Generally, even if $\operatorname{Re}_{\mathrm{m}} \gg 1$, the toroidal field is comparable to the radial one if $a_{\mathrm{e}} \sim \mathrm{Re}_{\mathrm{m}}$ despite a strong differential rotation. It is possible, for example, that the toroidal and poloidal fields measured in FU Orionis are comparable because of the Hall effect.

We consider the stability of axisymmetric short-wavelength perturbations. Small perturbations will be indicated by subscript 1 , while unperturbed quantities will have no subscript. The linearized momentum equation is:

$$
\begin{aligned}
\dot{\boldsymbol{v}}_{1}+\left(\boldsymbol{v}_{1} \cdot \nabla\right) \boldsymbol{v}+(\boldsymbol{v} \cdot \nabla) \boldsymbol{v}_{1} & =-\frac{\nabla p_{1}}{\rho}+\frac{\rho_{1}}{\rho}\left[\frac{\nabla p}{\rho}\right. \\
\left.-\frac{1}{4 \pi \rho}(\nabla \times \boldsymbol{B}) \times \boldsymbol{B}\right] & +\frac{1}{4 \pi \rho}\left(\nabla \times \boldsymbol{B}_{1}\right) \times \boldsymbol{B} \\
& +\frac{1}{4 \pi \rho}(\nabla \times \boldsymbol{B}) \times \boldsymbol{B}_{1} .
\end{aligned}
$$

Taking into account that the unperturbed motion is rotation $(\boldsymbol{v}=$ $s \Omega \boldsymbol{e}_{\varphi}$ ) and using Eq. (10), we can transform this equation into

$$
\begin{aligned}
\dot{\boldsymbol{v}}_{1}+ & 2 \Omega \times \boldsymbol{v}_{1}+\boldsymbol{e}_{\varphi} s \Omega^{\prime} v_{1 s}=-\frac{\nabla p_{1}}{\rho}+\frac{\rho_{1}}{\rho} \boldsymbol{D} \\
& +\frac{1}{4 \pi \rho}\left[\left(\nabla \times \boldsymbol{B}_{1}\right) \times \boldsymbol{B}+(\nabla \times \boldsymbol{B}) \times \boldsymbol{B}_{1}\right] .
\end{aligned}
$$

The term proportional to $\rho_{1} / \rho$ is typically small in a shortwavelength approximation. Indeed, we can estimate $p_{1} \sim c_{\mathrm{s}}^{2} \rho_{1}$ where $c_{\mathrm{s}}$ is the sound speed. Then, the pressure term in Eq. (16) is $\left(c_{\mathrm{s}}^{2} / \lambda\right)\left(\rho_{1} / \rho\right)$ where $\lambda$ is the lengthscale of perturbations. Observations indicate that the angular velocity can be smaller than the Keplerian angular velocity at least in a fraction of the disk volume (Donati et al. 2005) and, hence, $D \sim g$. Note that even departures by a factor of 2-3 from the Keplerian velocity reported by Donati et al. (2005) can produce a substantial increase in the local disk thickness compared to the "canonical" value $\sim 0.1 \mathrm{~s}$. Comparing the first two terms on the r.h.s. of Eq. (16), we conclude that the pressure term is larger if $c_{\mathrm{s}}^{2} / \lambda \gg D \sim g$, or $\lambda \ll c_{\mathrm{s}}^{2} / g$. Since $c_{\mathrm{s}}^{2} / g$ is the pressure scaleheight, this inequality is equivalent to the condition of applicability of a short wavelength approximation. Therefore, we can neglect the term proportional to $\boldsymbol{D}$ in what follows. An additional simplification is related to the last term on the r.h.s. of Eq. (16) (the "curvature term" according to Pessah \& Psaltis 2005). The toroidal field can often be stronger than the poloidal one and, as a result, the influence of unperturbed electric currents can be important. However, if the toroidal field satisfies the condition:

$B_{\varphi}<(s / \lambda) \max \left(B_{\mathrm{s}}, B_{z}\right)$

the contribution of "the curvature term" is small and can be neglected.

Following similar transformations with the remaining Eqs. (6)-(9), we arrived at the linearized equations needed for stability analysis. We consider perturbations with the space-time 
dependence $\propto \exp (\sigma t-\mathrm{i} \boldsymbol{k} \cdot \boldsymbol{r})$, where $\boldsymbol{k}=\left(k_{\mathrm{s}}, 0, k_{z}\right)$ is the wavevector. Then, the linearized MHD-equations read with accuracy in the lowest order in $\lambda / s$ :

$$
\begin{gathered}
\sigma \boldsymbol{v}_{1}+2 \boldsymbol{\Omega} \times \boldsymbol{v}_{1}+\boldsymbol{e}_{\varphi} s \Omega^{\prime} v_{1 \mathrm{~s}}=\frac{\mathrm{i} \boldsymbol{k} p_{1}}{\rho} \\
-\frac{i}{4 \pi \rho}\left(\boldsymbol{k} \times \boldsymbol{B}_{1}\right) \times \boldsymbol{B}, \\
\sigma \rho_{1}-\mathrm{i} \rho\left(\boldsymbol{k} \cdot \boldsymbol{v}_{1}\right)=0, \\
\sigma p_{1}-\mathrm{i} \gamma p\left(\boldsymbol{k} \cdot \boldsymbol{v}_{1}\right)=0,
\end{gathered}
$$

$$
\begin{array}{r}
\left(\sigma+\omega_{\eta}\right) \boldsymbol{B}_{1}=\boldsymbol{e}_{\varphi} s \Omega^{\prime} B_{1 \mathrm{~s}}-\mathrm{i}(\boldsymbol{B} \cdot \boldsymbol{k}) \boldsymbol{v}_{1}+i \boldsymbol{B}\left(\boldsymbol{k} \cdot \boldsymbol{v}_{1}\right) \\
-\frac{c(\boldsymbol{k} \cdot \boldsymbol{B})}{4 \pi e n_{\mathrm{e}}} \boldsymbol{k} \times \boldsymbol{B},
\end{array}
$$

$\boldsymbol{k} \cdot \boldsymbol{B}_{1}=0$,

where $\omega_{\eta}=\eta k^{2}$.

The dispersion equation, corresponding to Eqs. (18)-(22) is rather complex in the general case. Therefore, we consider only a particular case when the wavevector is perpendicular to the magnetic field, $\boldsymbol{k} \cdot \boldsymbol{B}=0$. After some algebra, Eqs. (18)-(22) can be combined into a sixth-order dispersion relation,

$\sigma^{6}+a_{5} \sigma^{5}+a_{4} \sigma^{4}+a_{3} \sigma^{3}+a_{2} \sigma^{2}+a_{1} \sigma+a_{0}=0$.

The coefficients of this equation are expressed in terms of characteristic frequencies,

$a_{5}=2 \omega_{\eta}, \quad a_{4}=\omega_{\eta}^{2}+\omega_{0}^{2}+\kappa^{2}$,

$a_{3}=\omega_{B \Omega}^{3}+\omega_{\eta}\left(\omega_{0}^{2}+\omega_{\mathrm{s}}^{2}+2 \kappa^{2}\right)$,

$a_{2}=\omega_{\eta}^{2}\left(\omega_{\mathrm{s}}^{2}+\kappa^{2}\right)+\mu \kappa^{2} \omega_{0}^{2}$,

$a_{1}=\mu \kappa^{2}\left[\omega_{B \Omega}^{3}+\omega_{\eta}\left(\omega_{\mathrm{s}}^{2}+\omega_{0}^{2}\right)\right], a_{0}=\mu \omega_{\mathrm{s}}^{2} \omega_{\eta}^{2} \kappa^{2}$,

where $\mu=k_{z}^{2} / k^{2}$. The characteristic frequences are given by

$\kappa^{2}=4 \Omega^{2}+2 s \Omega \Omega^{\prime}, \quad \omega_{0}^{2}=\omega_{\mathrm{s}}^{2}+\omega_{\mathrm{m}}^{2}, \quad \omega_{\mathrm{s}}^{2}=c_{\mathrm{s}}^{2} k^{2}$,

$\omega_{\mathrm{m}}^{2}=c_{\mathrm{A}}^{2} k^{2}, \quad \boldsymbol{c}_{\mathrm{A}}=\boldsymbol{B} / \sqrt{4 \pi \rho}, \quad \omega_{B \Omega}^{3}=k^{2} c_{\mathrm{A} \varphi} c_{\mathrm{As}} s \Omega^{\prime}$,

where $c_{\mathrm{s}}=\sqrt{\gamma p / \rho}$ is the sound speed. Equation (23) describes six modes that can generally exist in a compressible, rotating, magnetized gas.

In the non-dissipative, incompressible, limit when the sound speed $c_{\mathrm{S}}$ is very large, Eq. (23) reduces to the dispersion relation for the inertial waves,

$\sigma^{2}\left(\sigma^{2}+\mu \kappa^{2}\right)=0$.

This equation allows for unstable solutions if the Rayleigh criterion is fulfilled, $\kappa^{2}<0$. Note that Eq. (23) does not describe the magnetorotational instability since it does not occur for perturbations with $\boldsymbol{k} \cdot \boldsymbol{B}=0$.

In non-dissipative limit, $\eta \rightarrow 0$, Eq. (23) recovers the dispersion relation derived by Bonanno \& Urpin (2006) for perturbations with $\boldsymbol{k} \cdot \boldsymbol{B}=0$,

$\sigma^{5}+\sigma^{3}\left(\omega_{0}^{2}+\kappa^{2}\right)+\sigma^{2} \omega_{B \Omega}^{3}+\sigma \mu \kappa^{2} \omega_{0}^{2}+\mu \kappa^{2} \omega_{B \Omega}^{3}=0$.

The authors argued that Eq. (25) has unstable solutions even when the criteria of the magnetorotational and Rayleigh instability are not satisfied. The condition of instability is $\omega_{B \Omega} \neq 0$ and, hence, the shear-driven instability arises if $B_{\mathrm{s}} \neq 0$. The paper by
Bonanno \& Urpin (2007) considers the dispersion relation for any wavevectors, but does so in a high-conductivity limit $\eta \rightarrow 0$.

Equation (23) can be simplified in many cases of interest if we take into account that, likely, the $s$-component of the magnetic field in disks is greater than the z-component, $B_{\mathrm{s}} \gg B_{z}$. For perturbations with $\boldsymbol{k} \cdot \boldsymbol{B}=0$, we have $k_{\mathrm{s}}=-k_{z} B_{z} / B_{\mathrm{s}}$. Then,

$\mu=\frac{k_{z}^{2}}{k_{z}^{2}+k_{\mathrm{s}}^{2}}=\frac{B_{\mathrm{s}}^{2}}{B_{\mathrm{s}}^{2}+B_{z}^{2}} \approx 1$.

Substituting $\mu \approx 1$ into Eq. (23), we can transform it into

$$
\begin{aligned}
& \left(\sigma^{2}+\kappa^{2}\right)\left[\sigma^{4}+2 \omega_{\eta} \sigma^{3}+\left(\omega_{\eta}^{2}+\omega_{0}^{2}\right) \sigma^{2}\right. \\
& \left.+\left(\omega_{\eta} \omega_{\mathrm{s}}^{2}+\omega_{\eta} \omega_{0}^{2}+\omega_{B \Omega}^{3}\right) \sigma+\omega_{\eta}^{2} \omega_{\mathrm{s}}^{2}\right]=0
\end{aligned}
$$

Two roots of this equation describe the inertial waves which can be unstable only if the Rayleigh criterion is satisfied. Other four modes are described by the dispersion relation

$$
\begin{array}{r}
\sigma^{4}+2 \omega_{\eta} \sigma^{3}+\left(\omega_{\eta}^{2}+\omega_{0}^{2}\right) \sigma^{2}+\left(\omega_{\eta} \omega_{\mathrm{s}}^{2}+\omega_{\eta} \omega_{0}^{2}+\omega_{B \Omega}^{3}\right) \sigma \\
+\omega_{\eta}^{2} \omega_{\mathrm{s}}^{2}=0 .
\end{array}
$$

This equation describes fast and slow magnetoacoustic waves, and we consider the stability of these modes. Note that simplification (26) is made for mathematical convenience rather than for physical relevance. In fact, the dispersion relation (28) for fast and slow magnetosonic waves will change little if $\mu \neq 1$. This particularly concerns the modes with $|\sigma|>\Omega$ because the dispersion equation for them will differ from Eq. (28) only by terms of the order of $(\mu-1) \kappa^{2} / \sigma^{2}$ in coefficients. Therefore, our results can be applied with a sufficient accuracy also for the perturbations with $\mu \neq 1$.

\section{Criteria of instability in protostellar disks}

The Hurwitz theorem states that an equation of the fourth order,

$\sigma^{4}+b_{3} \sigma^{3}+b_{2} \sigma^{2}+b_{1} \sigma^{1}+b_{0}=0$

has at least one root with a positive real part (unstable mode) if one of the following inequalities

$b_{3}<0, \quad b_{0}<0$,

$b_{3} b_{2}-b_{1}<0$

$b_{1}\left(b_{3} b_{2}-b_{1}\right)-b_{3}^{2} b_{0}<0$,

is fulfilled (see Aleksandrov et al. 1985). Conditions (30) are never satisfied in protostellar disks. Therefore, the instability arises if one of the inequalities (31) or (32) are satisfied. These inequalities can be rewritten for Eq. (28) as

$\omega_{\eta}\left(2 \omega_{\eta}^{2}+\omega_{\mathrm{m}}^{2}\right)-\omega_{B \Omega}^{3}<0$,

$\omega_{B \Omega}^{6}-2 \omega_{B \Omega}^{3} \omega_{\eta}\left(\omega_{\eta}^{2}-\omega_{\mathrm{s}}^{2}\right)-\omega_{\eta}^{2} \omega_{\mathrm{m}}^{2}\left(\omega_{0}^{2}+2 \omega_{\eta}^{2}+\omega_{\mathrm{s}}^{2}\right)>0$.

Generally, the expressions on the 1.h.s. of both these inequalities can have a positive or negative sign, depending on the value of $\omega_{B \Omega}$. Therefore, Eqs. (33) and (34) impose restrictions on the rate of differential rotation which should be greater than some critical value. Note that, if the azimuthal field is generated by winding up the radial field, then $B_{\mathrm{s}} B_{\varphi} \Omega^{\prime}>0$ and, hence, $\omega_{B \Omega}^{3}>$ 0 as well. Therefore, we consider only this case. Denoting $\delta=$ $c_{\mathrm{As}} c_{\mathrm{A} \varphi} / c_{\mathrm{A}}^{2}$, we have from Eq. (33)

$\delta \frac{H s \Omega^{\prime}}{c_{\mathrm{A}}}>\frac{\eta}{c_{\mathrm{A}} H}\left[\frac{2 \eta^{2}}{c_{\mathrm{A}}^{2} H^{2}}(k H)^{4}+(k H)^{2}\right]$. 


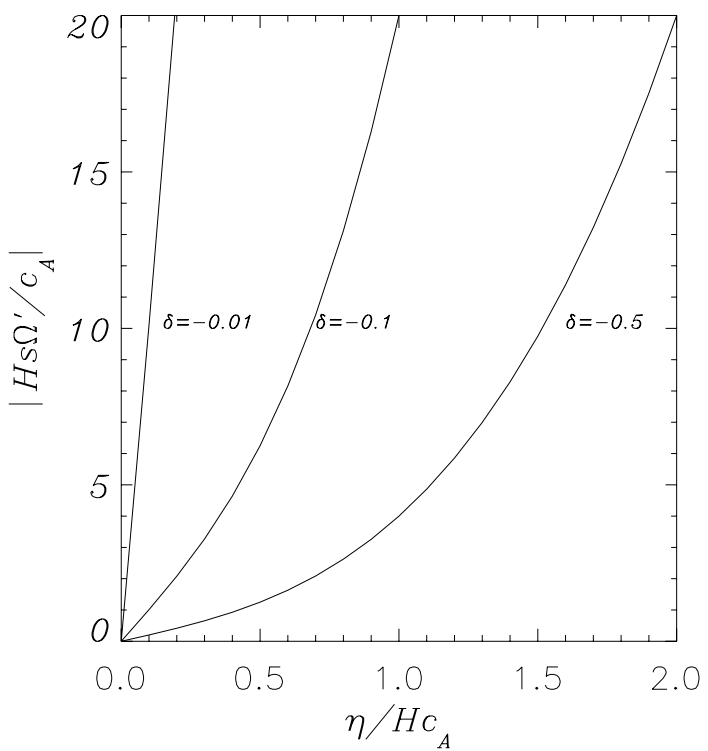

Fig. 1. The region of parameters where the instability can occur in accordance with Eq. (32) for different values $\delta$.

This condition can be satisfied only if the differential rotation is sufficiently strong. Since $\Omega^{\prime}<0$ in astrophysical disks, we consider only this case. Then, we have $\delta<0$ because $B_{\mathrm{s}} B_{\varphi} \Omega^{\prime} \propto$ $\delta \Omega^{\prime}>0$. The r.h.s. of Eq. (35) is a monotonously increasing function of $k H$ and is a minimum for the minimal possible value of $k H$. Since a short-wavelength approximation applies only if $k H>1$, we can estimate the domain of instability supposing $k H=1$ in inequality (35). Then, in order to fulfill the instability condition (35) for short-wavelength perturbations with $k H>1$, the rotation shear has to satisfy, at least, the inequality:

$\delta \frac{H s \Omega^{\prime}}{c_{\mathrm{A}}}>\frac{\eta}{c_{\mathrm{A}} H}\left(\frac{2 \eta^{2}}{c_{\mathrm{A}}^{2} H^{2}}+1\right)$.

In Fig. 1, we plot the regions of instability given by Eq. (36) for different negative values of $\delta$. At given $\delta$, the instability can occur if the value of $\left|H s \Omega^{\prime} / c_{\mathrm{A}}\right|$ is above the corresponding curve (note that $\Omega^{\prime}$ is negative in Fig. 1). If the magnetic and thermal energies are comparable and departures from the Keplerian disk are not large, then $c_{\mathrm{A}} / H \sim c_{\mathrm{s}} / H \sim \Omega$, and $H s \Omega^{\prime} / c_{\mathrm{s}} \sim$ $s \Omega^{\prime} / \Omega \approx 3 / 2$. Inequality (36) can be satisfied only for relatively small values of $\eta / H c_{\mathrm{A}}<|\delta|$, or $|\delta| \sim B_{\mathrm{s}} / B_{\varphi}>1 / \mathrm{Re}_{\mathrm{m}}$. This condition can be satisfied only if the Hall parameter is large $a_{\mathrm{e}}>1$, because $\delta \sim 1 / \operatorname{Re}_{\mathrm{m}}$ (see Eq. (13)) in the case $a_{\mathrm{e}}<1$. Condition (36) can also be fulfilled in a non-Keplerian disk. For a non-Keplerian disk, the quantity $H s \Omega^{\prime} / c_{\mathrm{A}}$ can vary in a wider range even if the magnetic and thermal energies are comparable, but condition (36) still cannot be satisfied for relatively large values of $\eta / H c_{\mathrm{A}}$.

By analogy, we can also transform condition (34). The minimal unstable rotation shear corresponds again to the maximal possible wavelength. Again, the maximum wavelength can be estimated from the condition $k H \sim 1$. For such wavevectors, Eq. (36) can be transformed into:

$$
\begin{array}{r}
\delta^{2}\left(\frac{H s \Omega^{\prime}}{c_{\mathrm{A}}}\right)^{2}>2 \delta\left(\frac{H s \Omega^{\prime}}{c_{\mathrm{A}}}\right) \frac{\eta}{H c_{\mathrm{A}}}\left(\frac{\eta^{2}}{H^{2} c_{\mathrm{A}}^{2}}-\beta\right) \\
+\frac{\eta^{2}}{H^{2} c_{\mathrm{A}}^{2}}\left(1+2 \beta+\frac{2 \eta^{2}}{H^{2} c_{\mathrm{A}}^{2}}\right),
\end{array}
$$

where $\beta=c_{\mathrm{s}}^{2} / c_{\mathrm{A}}^{2}$.

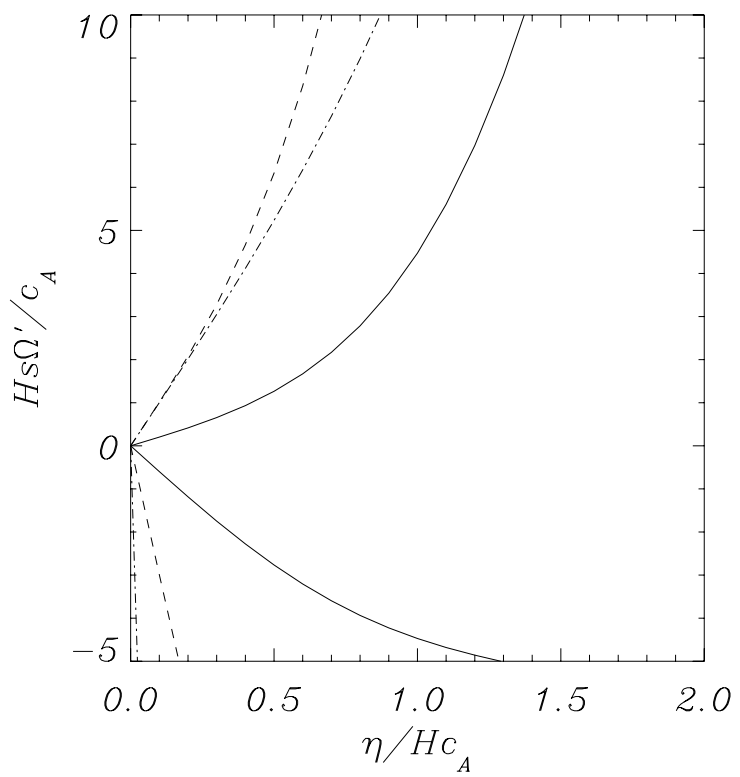

Fig. 2. The region of parameters where instability can occur in accordance with Eq. (33). The values of $[\delta, \beta]$ are $[-0.5,1]$ (solid lines), $[-0.1,1]$ (dashed lines), and $[-0.1,10]$ (dashed-and-dotted lines).

In Fig. 2, we show the region of parameters where the instability can occur in accordance with Eq. (37). Inequality (37) is quadratic in $H s \Omega^{\prime} / c_{\mathrm{A}}$ and can be satisfied if this parameter is larger/smaller than the largest/smallest root of the corresponding quadratic equation. Therefore, for a given combination of $\beta$ and $\delta$, the regions of instability lie above the upper line and below the lower line.

\section{Growth rate of instability}

To calculate the instability growth rate, it is convenient to introduce dimensionless quantities,

$$
\begin{array}{r}
\Gamma=\frac{\sigma}{\Omega}, \quad \alpha=\frac{\eta}{\Omega H^{2}}, \quad \varepsilon=\frac{c_{\mathrm{s}}}{H \Omega}, \quad x=k H, \\
\delta=\frac{c_{A \phi} c_{\mathrm{As}}}{c_{\mathrm{A}}^{2}}, \quad q=\left|\frac{s \Omega^{\prime}}{\Omega}\right|,
\end{array}
$$

(we assume $B_{\mathrm{s}} B_{\varphi} \Omega^{\prime}>0$ ). Then, Eq. (24) transforms into

$$
\begin{aligned}
& \Gamma^{4}+2 \alpha x^{2} \Gamma^{3}++\Gamma^{2} x^{2}\left[\alpha^{2} x^{2}+\varepsilon^{2}(1+1 / \beta)\right] \\
& +\Gamma \varepsilon^{2} x^{2}\left[\alpha x^{2}(2+1 / \beta)+q \delta / \beta\right]+\alpha^{2} \varepsilon^{2} x^{6}=0 .
\end{aligned}
$$

The dependence on the wavelength is characterized by the parameter $x^{2}$ in this equation. The parameter $\alpha$ is of the order of $1 / \mathrm{Re}_{\mathrm{m}}$. We solved Eq. (38) numerically for different values of the parameters by computing the eigenvalues of the matrix whose characteristic polynomial is given by Eq. (38) (see Press et al. 1992, for details).

In Fig. 3, we plot the dependence of the growth rate on $x$ for the Keplerian disk with $q=3 / 2$ and $\varepsilon=1$ and for several values of the parameter $\beta$. In all cases, the growth rate decreases monotonically with decreasing wavelength $\lambda=2 \pi / k$ because ohmic dissipation is more efficient for perturbations with a shorter wavelength. For any ratio of the magnetic and gas pressures, the instability does not occur if $k H>12$. If the magnetic pressure is greater than the gas pressure $(\beta<1)$, the growth rate can reach relatively large values $\Gamma \approx 0.04-0.06$. This corresponds to the growth time only 3-4 times longer than the rotation period. The magnetorotational instability cannot arise in 


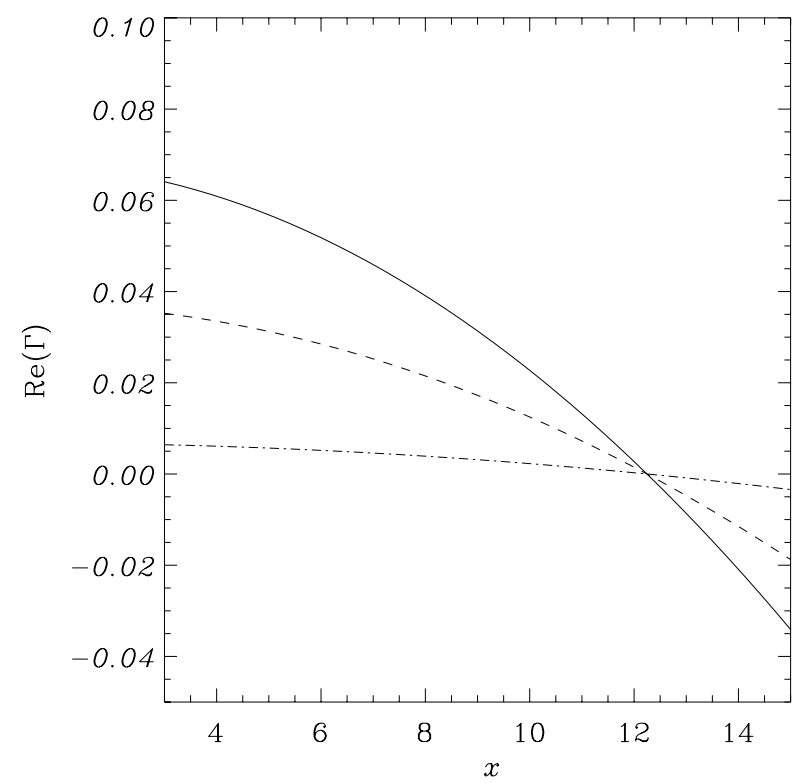

Fig. 3. The dependence of the growth rate on $x=k H$ for the Keplerian disk, with $q=3 / 2$ and $\varepsilon=1$, and for $\beta=0.1$ (solid line), 1 (dashed line), and 10 (dashed-and-dotted line). The other parameters are $\delta=0.1$ and $\alpha=0.001$.

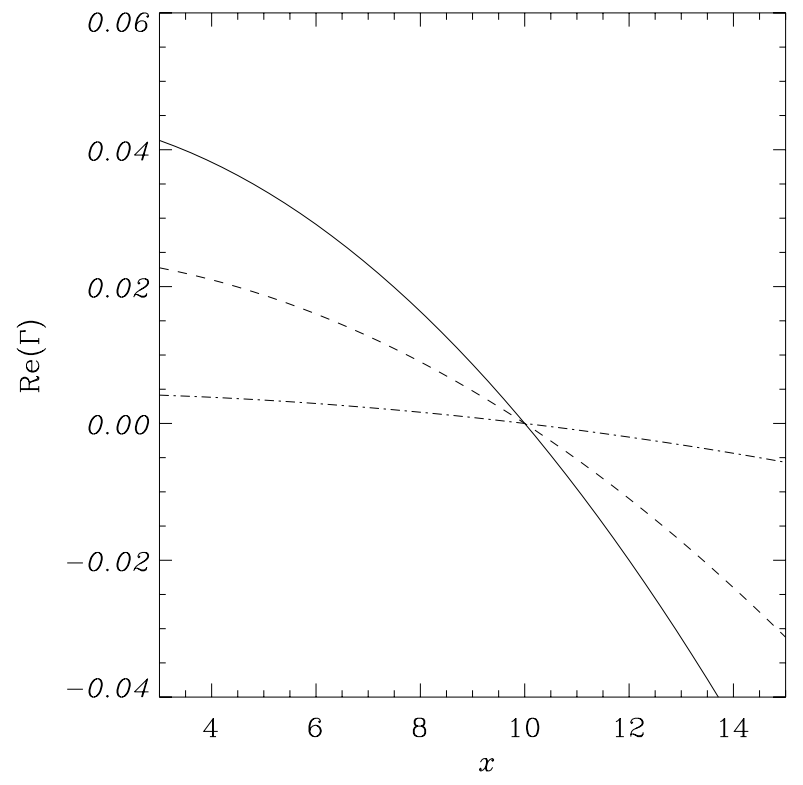

Fig. 4. The same as in Fig. 3 but for a non-Keplerian disk with $q=1$ and $\varepsilon=3$.

such a strong magnetic field no matter how large is the electrical conductivity of gas. Even if $\boldsymbol{k}$ is not perpendicular to $\boldsymbol{B}$, the MRI can occur only if the magnetic field and wavevector satisfy the condition $k^{2} c_{\mathrm{A}}^{2}<2 s \Omega \Omega^{\prime}$. Assuming that $s \Omega^{\prime} \sim \Omega$, we can transform this inequality approximately into $\Omega>c_{\mathrm{A}} k \sim x c_{\mathrm{S}} / H \sqrt{\beta}$. Since $c_{\mathrm{s}} / H \sim \Omega$ in the Keplerian disk, we find that MRI arises if $x / \sqrt{\beta}<1$. In the case $\beta<1$, this condition never applies and the MRI is suppressed. On the contrary, the shear-driven instability turns out to be rather efficient in such a strong field.

In Fig. 4, we plot the same dependence, but for the nonKeplerian disk with $q=1$ and $\varepsilon=3$. The other parameters are same. Qualitatively, the dependences are very similar to those shown in Fig. 3. The instability grows faster in the disk with larger $\beta$, which seems to be a general feature of the shear-driven

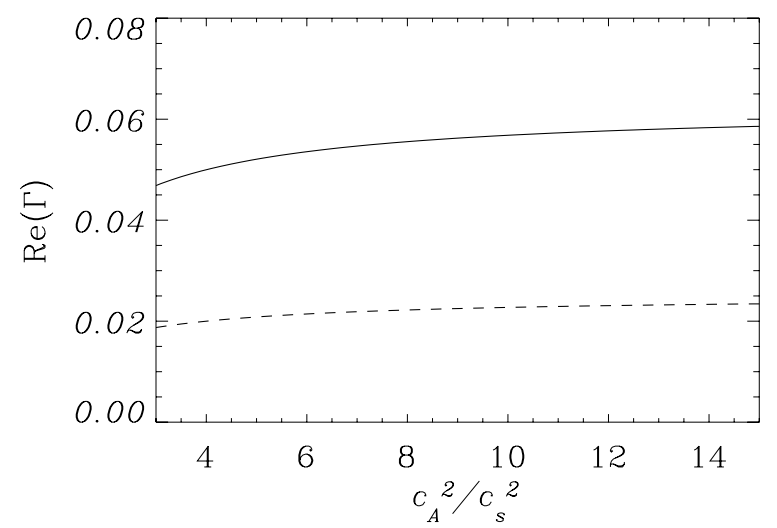

Fig. 5. The dependence of the growth rate on $\beta$ for the Keplerian disk with $q=3 / 2, \varepsilon=1, \delta=0.1$, and $\alpha=0.001$. The solid and dashed lines correspond to $x=5$ and 10 , respectively.

instability. However, the growth rate is typically smaller for a non-Keplerian disk. The range of unstable wavevectors is limited approximately by $k H<10$. Note that the half-thickness $H$ is essentially larger in a non-Keplerian disk than in the Keplerian one.

The dependence of the growth rate on $\beta^{-1}=c_{\mathrm{A}}^{2} / c_{\mathrm{S}}^{2}$ is shown in Fig. 5 for the disk with $q=3 / 2$ and $\varepsilon=1$. At a given wavelength, the growth rate increases with $1 / \beta$ slowly approaching a saturation value that depends on $q$. The saturation growth rate is $\sim 0.06 \Omega$ and $0.025 \Omega$ for $q=5$ and 10 , respectively. This correspond to the growth time of about $\sim 3$ and $\sim 6$ rotation periods. The dependence of the shear-driven instability on the magnetic field is unusual, at least, in the domain of parameters because usually a strong magnetic field tends to suppress other types of instability. Figure 6 shows the dependence of the growth rate on the parameter $\alpha$, which is approximately equal to the inverse magnetic Reynolds number, $\alpha \sim 1 / \mathrm{Re}_{\mathrm{m}}$. Therefore, higher values of $\alpha$ correspond to a higher dissipation rate, and the instability should be suppressed at large $\alpha$. Indeed, the growth rate goes to 0 if $\alpha \sim 0.001-0.01$, depending on the wavelength. Hence, one can expect that the shear-driven instability occurs even in disks with a relatively small magnetic Reynolds number $\sim 100$.

\section{Discussion}

We have considered the new instability that can arise in protostellar disks under the combined influence of the magnetic field, differential rotation, and compressibility. To illustrate the main qualitative features of the instability, we analyzed a particular case of perturbations with the wavevector $\boldsymbol{k}$ perpendicular to the magnetic field $\boldsymbol{B}$. In this case, the standard MRI does not occur because its growth rate is $\propto(\boldsymbol{k} \cdot \boldsymbol{B})$. The considered instability is related to shear and compressibility of a magnetized gas. In the incompressible limit $\left(c_{\mathrm{s}} \rightarrow \infty\right)$ we have, from Eq. (27),

$\left(\sigma^{2}+\kappa^{2}\right)\left(\sigma+\omega_{\eta}\right)^{2}=0$

and the instability does not occur. This is a major difference from other instabilities caused by differential rotation, such as the Rayleigh or magnetorotational instabilities that can occur in incompressible limit.

The magnetic field is likely thermal or superthermal in protostellar disks (see Donati et al. 2005). The considered instability can arise even in a very strong magnetic field when the MRI is suppressed completely, It is known that the MRI does not occur if the magnetic field satisfies the condition 


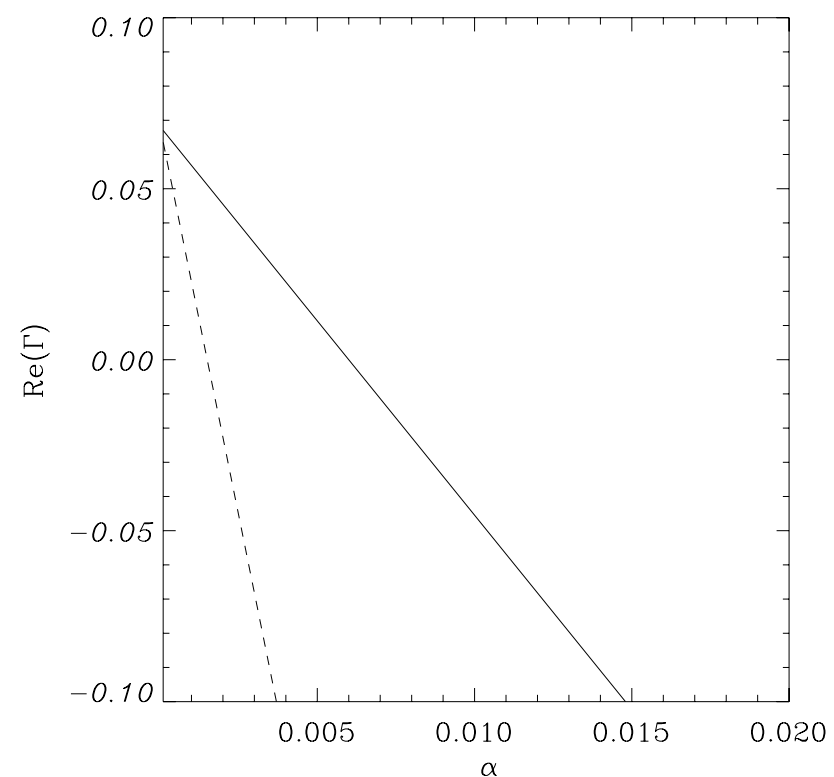

Fig. 6. The dependence of the growth rate on $\alpha$ for $q=3 / 2, \varepsilon=1$, $\delta=0.1$, and $\beta=0.1$. The solid and dashed curves correspond to $q=5$ and 10 , respectively.

$B>B_{\mathrm{cr}}=\left(s \Omega^{\prime} / k\right) \sqrt{4 \pi \rho}$. The shear-driven instability can operate even if the field is stronger than $B_{\mathrm{cr}}$. The growth time of instability is still rather short (several rotation periods) in the gas where the magnetic pressure is greater than the thermal pressure.

The criteria for the considered instability can be satisfied in protostellar disks. Likely, all disks have both radial and azimuthal magnetic fields. These magnetic fields are found in observations, as well as in numerical simulations. Superthermal magnetic fields do not suppress the instability crucial in cold protostellar disks. The instability can arise even in a low conductive gas where the magnetic Reynolds number associated with rotation is relatively small, $\operatorname{Re}_{\mathrm{m}} \sim 100$.

Our paper considers only a local axisymmetric instability. Most likely, the same mechanism of instability will efficiently destabilize perturbations with the wavelength comparable to the disk scale height. Dissipative effects are less important for these perturbations, and the unstable modes can grow even faster. A global instability caused by a combined influence of differential rotation and compressibility will be considered elsewhere. The turbulence that could be generated by the considered instability can be strongly anisotropic in the $(s, z)$-plane because both the criteria and growth rate are sensitive to the direction of the wave vector. The generated turbulence, however, might be efficient in the radial transport of angular momentum in strongly magnetized protostellar disks.

Acknowledgements. This research project has been supported by a Marie Curie Transfer of Knowledge Fellowship of the European Community's Sixth
Framework Programme under contract number MTKD-CT-002995. VU thanks also INAF-Osservatorio Astrofisico di Catania for hospitality.

\section{References}

Acheson, D. J. 1978, Phil. Trans. Roy. Soc. Lond. A, 289, 459

Acheson, D. J. 1979, Sol. Phys., 62, 23

Aleksandrov, A., et al. 1985, Mathematics: Its Content, Methods, and Meaning (Cambridge: MIT)

Arlt, R., \& Rüdiger, G. 2001, A\&A, 374, 1035

Arlt, R., \& Urpin, V. 2004, A\&A, 426, 755

Balbus, S. A., \& Hawley, J. F. 1991, ApJ, 376, 214

Balbus, S., \& Terquem, C. 2001, ApJ, 552, 235

Bonanno, A., \& Urpin, V. 2006, Phys. Rev. E, 73, 066301

Bonanno, A., \& Urpin, V. 2007, ApJ, 662, 851

Brandenburg, A., Nordlund, Å., Stein, R., \& Torkelsson, U. 1995, ApJ, 446, 741

Brandenburg, A., \& Rüdiger, G. 2005 [arXiv: astro-ph/0512409]

Donati, J.-F., Paletou, F., Bouvier, J., \& Ferreira, J. 2005, Nature, 438, 466

Draine, B., Roberge, W., \& Dalgarno, A. 1983, ApJ, 264, 485

Drecker, A., Rüdiger, G., \& Hollerbach, R. 2000, MNRAS, 317, 45

Dubrulle, B., Marié, L., Normand, Ch., et al. 2005, A\&A, 429, 1

Fleming, T., \& Stone, J. 2003, ApJ, 585, 908

Fricke, K. 1969, A\&A, 1, 388

Gammie, C. 1996, ApJ, 457, 355

Hawley, J. F., Gammie, C. F., \& Balbus, S. A. 1995, ApJ, 440, 742

Hutawarakorn, B., \& Cohen, R. J. 1999, MNRAS, 303, 845

Hutawarakorn, B., \& Cohen, R. J. 2005, MNRAS, 357, 338

Ji, H., Burin, M., Schartman, E., \& Goodman, J. 2006, Nature, 444, 343

Jin, L. 1996, ApJ, 457, 798

Johnston, B., \& Gammie, C. 2006, ApJ, 636, 63

Kaisig, M., Tajima, T., \& Lovelace, R. 1992, ApJ, 386, 83

Keppens, R., Casse, F., \& Goedbloed, J. 2002, ApJ, 569, L121

Klahr, N., \& Bodenheimer, P. 2003, ApJ, 582, 869

Kumar, S., Coleman, C., \& Kley, W. 1994, MNRAS, 266, 379

Lesur, G., \& Longaretti, P.-Y. 2005, A\&A, 444, 25

Livertz, E., Mond, M., \& Chernin, A. D. 2007 [arXiv: astro-ph/0701086]

Matsumoto, R., \& Tajima, T. 1995, ApJ, 445, 767

Pelletier, G., \& Pudritz, R. 1992, ApJ, 394, 117

Pessah, M., \& Psaltis, D. 2005, ApJ, 628, 879

Petersen, M., Stewart, G., \& Julien, K. 2007, ApJ, 658, 1252

Press, W. H., Teukolsky, S. A., Vetterling, W. T., \& Flannery, B. P. 1992, Numerical recipes in FORTRAN, The art of scientific computing (Cambridge: UP)

Rüdiger, G., \& Kitchatinov, L. L. 2005, A\&A, 434, 629

Safronov, V. 1969, Evolution of the protoplanetary cloud and formation of the Earth (Moscow: Nauka)

Salmeron, R., \& Wardle, M. 2005, MNRAS, 361, 45

Sano, T., \& Stone, J. 2002b, ApJ, 577, 534

Sano, T., Inutsuka, S. I., \& Miyama, S. M. 1998, ApJ, 506, L57

Shtemler, Y., Mond, M., \& Livertz, E. 2007 [arXiv: astro-ph/0701088]

Spitzer, L. 1978, Physical Processes in the Interstellar Medium (New York: Wiley)

Stone, J., \& Balbus, S. 1996, ApJ, 464, 364

Torkelsson, U., Brandenburg, A., Nordlund, A., \& Stein, R. 1996, Astrophys. Lett. Comm., 34, 383

Turner, N., Sano, T., \& Dziourkevitch, N. 2007, ApJ, 659, 729

Urpin, V. 2003, A\&A, 404, 397

Urpin, V., \& Rüdiger, G. 2005, A\&A, 437, 23

Velikhov, E. P. 1959, Sov. Phys. JETP, 9, 995

Wardle, M. 1999, MNRAS, 307, 849

Zhang, W., Diamond, P., \& Vishniac, E. 1994, ApJ, 420, 705 